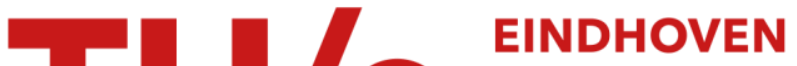 UNIVERSITY OF TECHNOLOGY
}

\section{Comparing exploration strategies for Q-learning in random stochastic mazes}

\section{Citation for published version (APA):}

Tijsma, A., Drugan, M. M., \& Wiering, M. A. (2017). Comparing exploration strategies for Q-learning in random stochastic mazes. In 2016 IEEE Symposium Series on Computational Intelligence (IEEE SSCI 2016) Proceedings, 6-9 December 2016, Athens, Greece [7849366] Institute of Electrical and Electronics Engineers. https://doi.org/10.1109/SSCI.2016.7849366

DOI:

10.1109/SSCI.2016.7849366

Document status and date:

Published: 13/02/2017

\section{Document Version:}

Accepted manuscript including changes made at the peer-review stage

\section{Please check the document version of this publication:}

- A submitted manuscript is the version of the article upon submission and before peer-review. There can be important differences between the submitted version and the official published version of record. People interested in the research are advised to contact the author for the final version of the publication, or visit the $\mathrm{DOI}$ to the publisher's website.

- The final author version and the galley proof are versions of the publication after peer review.

- The final published version features the final layout of the paper including the volume, issue and page numbers.

Link to publication

\section{General rights}

Copyright and moral rights for the publications made accessible in the public portal are retained by the authors and/or other copyright owners and it is a condition of accessing publications that users recognise and abide by the legal requirements associated with these rights.

- Users may download and print one copy of any publication from the public portal for the purpose of private study or research.

- You may not further distribute the material or use it for any profit-making activity or commercial gain

- You may freely distribute the URL identifying the publication in the public portal.

If the publication is distributed under the terms of Article 25fa of the Dutch Copyright Act, indicated by the "Taverne" license above, please follow below link for the End User Agreement:

www.tue.nl/taverne

Take down policy

If you believe that this document breaches copyright please contact us at:

openaccess@tue.nl

providing details and we will investigate your claim. 


\title{
Comparing Exploration Strategies for Q-learning in Random Stochastic Mazes
}

\author{
Arryon D. Tijsma*, Madalina M. Drugan ${ }^{\dagger}$ and Marco A. Wiering* \\ *Institute of Artificial Intelligence and Cognitive Engineering \\ University of Groningen \\ Email: a.d.tijsma@rug.nl,m.a.wiering@rug.nl \\ †Department of Mathematics and Computer Science \\ Technical University Eindhoven \\ Email: madalina.drugan@gmail.com
}

\begin{abstract}
Balancing the ratio between exploration and exploitation is an important problem in reinforcement learning. This paper evaluates four different exploration strategies combined with Q-learning using random stochastic mazes to investigate their performances. We will compare: UCB-1, softmax, $\epsilon$-greedy, and pursuit. For this purpose we adapted the UCB-1 and pursuit strategies to be used in the Q-learning algorithm. The mazes consist of a single optimal goal state and two suboptimal goal states that lie closer to the starting position of the agent, which makes efficient exploration an important part of the learning agent. Furthermore, we evaluate two different kinds of reward functions, a normalized one with rewards between 0 and 1 , and an unnormalized reward function that penalizes the agent for each step with a negative reward. We have performed an extensive grid-search to find the best parameters for each method and used the best parameters on novel randomly generated maze problems of different sizes. The results show that softmax exploration outperforms the other strategies, although it is harder to tune its temperature parameter. The worst performing exploration strategy is $\epsilon$-greedy.
\end{abstract}

\section{INTRODUCTION}

One of the most challenging tasks in reinforcement learning (RL) [1], [2] is that of balancing the ratio between exploration and exploitation. Too much exploration yields a lower accumulated reward, while too much exploitation can lead to the agent being stuck in a local optimum. This problem is known as the exploration/exploitation dilemma [3]-[5]. Because exploration may waste time exploring an irrelevant part of the environment, exploitation must happen simultaneously. One of the most widely used exploration strategies is $\epsilon$-greedy [6]. The advantage of this strategy is that it is not dependent on specific data such as counters [7] and as an allround exploration strategy, $\epsilon$-greedy is often hard to beat [8].

One can distinguish between two types of exploration: directed and undirected exploration [7], [9]. Undirected exploration is driven by randomness, the most trivial example being the 'random walk' [10] where the agent completely ignores the reward function and always performs random actions. $\epsilon$-greedy is another example of undirected exploration, and is a case of using semi-uniform distributions [11], as is action selection based on the utility of an action [6], [12], of which Boltzmann or softmax exploration is an example.
Directed exploration methods can be distinguished using three categories. Counter-based exploration [9], [13] keeps count of how many times a state-action pair has been visited, and evaluates actions based on a linear combination of an exploitation term and an exploration term. Another category relies on exploring parts of the environment where the errors were large during the last updates, so called errorbased exploration methods [14], [15]. Finally, there exists a category of directed exploration techniques called recencybased exploration techniques that prefer to select the action in a state that had not been selected for the longest time [9]. It has been shown that directed exploration strategies perform better than undirected strategies for solving particular difficult maze problems when model-based RL is used [9]. It has also been shown that for the multi-armed bandit problem [16], simple heuristics like undirected exploration sometimes outperform more advanced algorithms [17].

In multi-armed bandit problems, there exists an algorithm called UCB-1 (for Upper Confidence Bound), which is a smart directed counter-based exploration strategy that has been shown to perform well for multi-armed bandits [16]. In this paper, this strategy is transferred to a Markov decision process in a similar way as in [18]. In [18], the authors adapted discounted UCB-1-tuned to be combined with Q-learning. However, they did not compare this method to the exploration strategies we study in this paper.

Contributions. This paper contributes a new comparison of exploration strategies in a stochastic maze problem. We use Q-learning [6] as reinforcement learning algorithm and compare four different exploration strategies by examining their cumulative reward intake. For this purpose, we built a random stochastic maze generator in which there are one optimal goal state and two suboptimal goal states. The task of the agent is to learn to navigate to the optimal goal state using the least amount of steps. We adapted the UCB-1 and pursuit exploration strategies, often used for the multi-armed bandit problem, to Q-learning with state-action pairs. We compare UCB-1, $\epsilon$-greedy, softmax and pursuit as exploration strategies using two different reward functions, a normalized one with rewards between 0 and 1 , and an unnormalized reward function that penalizes every step not ending at a 
goal state with a negative value. We also explore the effect of optimistic initialization of the Q-values by comparing this initialization scheme to the use of initial Q-values of zero. Furthermore, heatmaps for the exploration strategies are computed to gain insight into the general behavior of the reward intake as a function of the learning rate and each strategy's tunable parameter. This is important because certain parameter optimization algorithms benefit from a structured search space [19], and this gives insight into how easy it is to tune a particular exploration method in combination with Q-learning. The best found parameters are tested on novel generated mazes of different sizes, to make it possible to investigate the direct use of the best found parameters on a smaller maze to a maze problem of a bigger size. Our research question is: when learning to navigate in a stochastic maze using different exploration strategies, which strategy yields the largest accumulated reward, and which is the most consistent over different parameter values?

Outline of this paper. Section II presents the principles of reinforcement learning, Markov Decision Processes (MDPs), Q-learning, and the details of the four exploration strategies. Section III describes the methods used for training and evaluating the performance of the different exploration strategies. Section IV shows the results obtained from examining the influence of the two tunable parameters for each method and we report the results of different tests with the optimal parameters for each exploration strategy. Finally, the conclusions are presented in Section V.

\section{REINFORCEMENT LEARNING}

Reinforcement learning [1], [2], [20] is an area of machine learning where an agent is connected to an environment via perception and action. At each step, the agent receives an input, chooses and executes an action, and this changes the state of the environment. The reward of executing the action in the previous state is then given to the agent by a reward function. The agent should maximize the cumulative sum of obtained rewards by choosing preferred actions, learned by trial and error using a particular reinforcement learning algorithm. The underlying model of this sequential decision making problem is a Markov Decision Process (MDP) [21], defined by the following principles:

- A discrete set of $n$ states $S=\left\{s^{1}, s^{2}, \ldots, s^{n}\right\}$, where $s_{t} \in S$ describes the state of the environment at time step $t$.

- A discrete set of $m$ actions $A=\left\{a^{1}, a^{2}, \ldots, a^{m}\right\}$, where $a_{t}$ denotes the action selected by the agent at time $t$.

- A transition function $T\left(s, a, s^{\prime}\right)$ that maps state-action pair $s, a$ to the next state $s^{\prime}$ with a given transition probability.

- A reward function $R\left(s, a, s^{\prime}\right)$ denotes the average reward the agent obtains when transiting from state $s$ to state $s^{\prime}$ using action $a . r_{t}$ is the reward obtained at time $t$.
- A discount factor $0 \leq \gamma \leq 1$ that assigns a higher importance to immediate rewards compared to future rewards.

In reinforcement learning, the agent uses its past experiences to learn the optimal policy $\pi^{*}$ which maps states to optimal actions that optimize the cumulative reward intake. Learning this policy involves the estimation of a value-function using past experiences, called the Q-function [1]. The Q-function $Q^{\pi}(s, a)$ denotes the expected accumulated discounted future reward obtained by selecting action $a$ in state $s$ and following policy $\pi$ afterwards:

$$
Q^{\pi}(s, a)=E\left(\sum_{t=1}^{\infty} \gamma^{t-1} r_{t} \mid s_{1}=s, a_{1}=a, \pi\right)
$$

Q-learning [6] is an off-policy temporal difference (TD) [22] learning technique. With an off-policy learning method, the agent follows a behavioral policy and at the same time learns about the optimal Q-function. If the agent visits all state-action pairs an infinite number of times, Q-learning converges to the optimal Q-function [23]. Therefore, Q-learning can be used to learn the optimal policy for a given MDP [24], [25]. When the optimal Q-function is known, the optimal policy selects the action with the highest Q-value in a state.

The Q-learning update rule of the Q-value of a state-action pair at time step $t+1, Q_{t+1}\left(s_{t}, a_{t}\right)$ after an experience $s_{t}, a_{t}, s_{t+1}, r_{t}$, is as follows:

$Q_{t+1}\left(s_{t}, a_{t}\right)=Q_{t}\left(s_{t}, a_{t}\right)+\alpha\left[r_{t}+\gamma \max _{a} Q_{t}\left(s_{t+1}, a\right)-Q_{t}\left(s_{t}, a_{t}\right)\right]$

Where $0 \leq \alpha \leq 1$ is the learning rate of the update rule.

\section{A. Exploration Strategies}

An essential part of Q-learning in finding an optimal policy is the selection of action $a_{t}$ in state $s_{t}$. In Q-learning, there exists a tradeoff between selecting the currently expected optimal action, or selecting a different action in the hope it will yield a higher cumulative reward in the future. To investigate the goal-finding abilities of an agent in a maze using Q-learning, we investigate four different exploration strategies: $\epsilon$-greedy, Boltzmann (also called softmax), pursuit, and UCB-1. We will discuss them below.

1) $\epsilon$-greedy: $\epsilon$-greedy exploration is one of the most used exploration strategies. It uses $0 \leq \epsilon \leq 1$ as parameter of exploration to decide which action to perform using $Q_{t}\left(s_{t}, a\right)$. The agent chooses the action with the highest Q-value in the current state with probability $1-\epsilon$, and a random action otherwise. A larger value for $\epsilon$ means more exploration actions are selected by the agent. Randomness is necessary for an agent navigating through a stochastic maze to learn the optimal policy. With the experiments, we will find out which value of $\epsilon$ is optimal for our maze setup and how $\epsilon$-greedy compares to other exploration strategies. 
2) Boltzmann (or softmax) exploration: One drawback of $\epsilon$-greedy exploration is that the exploration action is selected uniform randomly from the set of possible actions. Therefore, it is as likely to choose the worst appearing action as it is to choose the second-best appearing action if an exploration action is selected. That is why Boltzmann or softmax exploration [26] uses the Boltzmann distribution function to assign a probability $\pi\left(s_{t}, a\right)$ to the actions in order to create a graded function of estimated value:

$$
\pi\left(s_{t}, a\right)=\frac{e^{Q_{t}\left(s_{t}, a\right) / T}}{\sum_{i=1}^{m} e^{Q_{t}\left(s_{t}, a^{i}\right) / T}}
$$

$\pi\left(s_{t}, a\right)$ denotes the probability the agent selects action $a$ in state $s_{t}$ and $T \geq 0$ is the temperature parameter used in the Boltzmann distribution. When $T=0$ the agent does not explore at all, and when $T \rightarrow \infty$ the agent selects random actions. Using softmax exploration with intermediate values for $T$, the agent still most likely selects the best action, but other actions are ranked instead of randomly chosen.

3) Pursuit: The pursuit method is adapted from the multiarmed bandit problem [1]. A pursuit method maintains both action-value estimates and action preferences for the current state. Let $\pi_{t}\left(s_{t}, a\right)$ be the probability of selecting action $a$ when in state $s_{t}$. After each time step $t$ and the update of the Q-value, the greedy action $a_{t+1}^{*}=\arg \max _{a} Q_{t+1}\left(s_{t}, a\right)$ has a probability of successively being selected that is incremented with a fraction $\beta$ towards one:

$$
\pi_{t+1}\left(s_{t}, a_{t+1}^{*}\right)=\pi_{t}\left(s_{t}, a_{t+1}^{*}\right)+\beta\left[1-\pi_{t}\left(s_{t}, a_{t+1}^{*}\right)\right]
$$

The probabilities for all the other actions in state $s_{t}$, on the other hand, are decreased towards zero:

$\pi_{t+1}\left(s_{t}, a\right)=\pi_{t}\left(s_{t}, a\right)+\beta\left[0-\pi_{t}\left(s_{t}, a\right)\right]$, for all $a \neq a_{t+1}^{*}$

where $\beta>0$ denotes the learning rate for the action preferences.

4) $U C B-1$ : The exploration strategy $U C B-1$ was also proposed for the multi-armed bandit problem [16], and is being adapted here for use with Q-learning. The UCB-1 strategy keeps a count of the number of times an action $a$ is executed in state $s$, for all actions and all states. After selecting an action, the strategy increases the count of the action taken in a state by one. The UCB-1 strategy first selects all actions exactly one time when a state is visited, so their counters have been populated. Afterwards, UCB-1 calculates an exploration bonus using:

$$
\operatorname{bonus}\left(s_{t}, a^{i}\right)=100 \times C \times \sqrt{\left(2 \times \frac{\log N\left(s_{t}\right)}{N\left(s_{t}, a^{i}\right)}\right)}
$$

where $N\left(s_{t}\right)=\sum_{i} N\left(s_{t}, a^{i}\right)$ denotes how often an action has been selected in state $s_{t}$, and $N\left(s_{t}, a^{i}\right)$ counts the number of times action $a^{i}$ has been selected in state $s_{t}$. The bonus becomes larger when the fraction of counts for all actions versus the count for action $a^{i}$ increases. A larger value of $C$ increases exploration. When $C=0$ there is no exploration and usually $C$ is set to a value of 1 . In [16] and [18], the term 100 does not appear in the equation to compute the bonus. However, we found that when using the unnormalized reward function (see section III-C) this aided the strategy's performance. For action selection, the bonus is added to the Q-value, and the action with the highest value in state $s_{t}$ is selected:

$$
a_{t}=\arg \max _{a}\left(Q_{t}\left(s_{t}, a\right)+\operatorname{bonus}\left(s_{t}, a\right)\right)
$$

Now, we have described the different exploration strategies. In the next section, we will describe the experimental setup and then we present the experimental results.

\section{EXPERIMENTAL SETUP}

\section{A. Stochastic Mazes}

The agent walks around in a maze environment. Figure 1 shows a $10 \times 10$ maze, with obstacles (blocks) $B$ and three separate goals, two suboptimal goal states denoted $g$ and the optimal goal state $G$. Each goal defines an end state to the maze, and the agent should learn to navigate to the optimal goal state. The agent always starts in state $A$. The rewards in the maze problem are shown in Table I and are further explained in section III-C. Walking outside the maze boundaries or against a block $B$ keeps the agent in the state it was previously in.

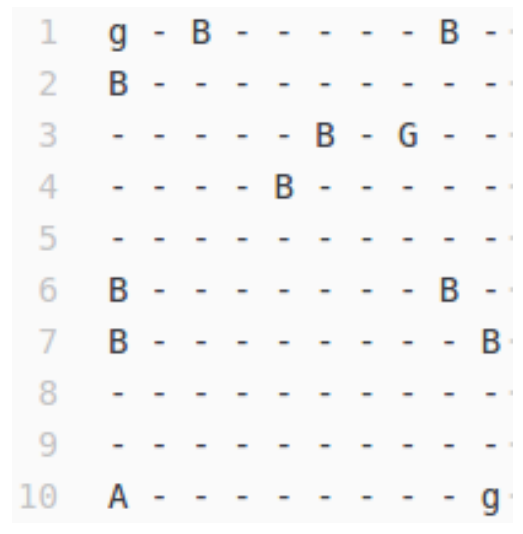

Fig. 1. A random $10 \times 10$ maze

TABLE I

REWARDS WITHIN THE STOCHASTIC MAZE

\begin{tabular}{rcc} 
& Unnormalized & Normalized \\
\hline step & -1 & 0 \\
\hline block (B) & -5 & 0 \\
\hline suboptimal goal $(\mathrm{g})$ & 50 & 0.5 \\
\hline optimal goal $(\mathrm{G})$ & 200 & 1.0 \\
\hline
\end{tabular}


We define a randomly generated maze by placing the agent and the goals always in the same places, but varying the placement of the blocks in the maze. In each maze, regardless of size, ten percent of the available spaces is occupied by blocks. Their positions are randomly sampled from the available spaces. In this way, the agent is forced to take a different route each time, but the absolute distances from the agent to the goals remain the same. A random maze is deemed solvable if the agent finds all goal states within 3000 steps when taking a random walk in the maze. We only use such solvable maze problems in the experiments.

\section{B. Terminology}

We will now explain the terminology used in the experiments:

- Epoch: a strategic walk through the maze until the agent reaches a goal state. Each epoch yields a cumulative reward and the total number of steps needed.

- Run: A full run of $k$ epochs for a given combination of parameters. A run yields a list of results containing the total cumulative reward and the total number of steps taken for each epoch.

- Search: One run for each combination of parameters, performed on a single maze. This yields the list of results for each parameter combination.

- Simulation: A search performed on each of the randomly generated mazes, yielding the results for each parameter combination per maze.

- Test: Using the best performing parameter combination from the simulation, we perform a run on each maze from a newly generated set of random mazes. The set of mazes can have different dimensions than the set on which we computed the best parameters for each method. The result is a list of results, which we average across runs to obtain a final score of performance for the strategy.

- Experiment: a set of simulations plus tests, each using different general parameters such as the initial Q-values of the Q-function, the rewards, or the transition probabilities.

\section{Normalized and Unnormalized Reward Functions}

In the original paper from Auer et al., the upper confidence bound of UCB-1 is proven given that the support of the rewards is $[0,1]$ [16]. This means that for correct comparison of the UCB-1 algorithm, we must also define a maze in which the reward is at most 1 , and at least 0 . We achieve this by defining two types of experiments using normalized and unnormalized reward functions. In the normalized version of the experiment, the support is defined within $[0,1]$ by assigning a reward of 1 to the optimal goal, a reward of 0.5 to the suboptimal goals, and removing the negative rewards from a step and a block-encounter, see also Table I.

Since we also want to study the use of optimistic initialization of the Q-values in each scenario, we ultimately define four different types of experiments:
1) $\neg \mathbf{O} / \neg \mathbf{N}$. Non-optimistic initialization, unnormalized reward function, where rewards are set according to column 1 in Table I and initial Q-values are set to zero.

2) $\mathbf{O} / \neg \mathbf{N}$. Optimistic initialization, unnormalized reward function, where rewards are set according to column 1 in Table I and initial Q-values are set to the value of the highest valued goal from column 1 .

3) $\neg \mathbf{O} / \mathbf{N}$. Non-optimistic initialization, normalized reward function, where rewards are set according to column 2 in Table I and initial Q-values are set to zero.

4) $\mathrm{O} / \mathrm{N}$. Optimistic initialization, normalized reward function, where rewards are set according to column 2 in Table I and initial Q-values are set to the value of the highest valued goal from column 2 .

\section{Experimental Setup}

As explained in Sections I and III-B, we perform an exhaustive search in the parameter space of each exploration strategy. Table III shows the range of parameters over which a search for each experiment is performed. The number of linearly spaced intervals for both $\alpha$ and the dependent parameter of each exploration strategy are set to 15 , resulting in 225 parameter combinations for each search. The low values for $C$ in UCB-1 in the normalized experiments is because we multiply this value by 100 when using the strategy, see Equation 4.

TABLE II

GRID SEARCH PARAMETERS USED IN NORMALIZED AND UNNORMALIZED REWARD FUNCTION EXPERIMENTS. NORM. = NORMALIZED

\begin{tabular}{rr|cc|cc|ccc|cc} 
& & \multicolumn{2}{c}{ UCB-1 } & \multicolumn{2}{c}{$\epsilon$-greedy } & \multicolumn{2}{c}{ softmax } & \multicolumn{2}{c}{ pursuit } \\
\hline norm. & & $\mathrm{C}$ & $\alpha$ & $\epsilon$ & $\alpha$ & $\mathrm{T}$ & $\alpha$ & $\beta$ & $\alpha$ \\
\hline \multirow{2}{*}{ no } & low & .2 & .1 & 0.0 & .1 & .5 & .1 & $1 \mathrm{e}-5$ & .1 \\
& high & 2.5 & .95 & 1.0 & .95 & 15 & .95 & .1 & .95 \\
\hline \multirow{2}{*}{ yes } & low & .002 & .1 & 0.0 & .1 & .01 & .1 & $1 \mathrm{e}-5$ & .1 \\
& high & .025 & .95 & 1.0 & .95 & 1.5 & .95 & .1 & .95 \\
\hline
\end{tabular}

In all simulations, the discount factor remains fixed at a value of $\gamma=0.98$, and the total number of epochs is set to 3000. Furthermore, the transition probabilities also remain the same. There is a $70 \%$ probability the agent ends up in the state resulting from the chosen action (North, West, South, East), and there is a $10 \%$ probability the agent goes to each of the other three adjacent states.

For each experiment and each simulation, we use the same set of ten random mazes of size $10 \times 10$ that we generated beforehand. For each test, we generated two new sets of ten mazes with sizes $10 \times 10$ and $20 \times 20$.

\section{RESULTS}

For each simulation, we calculate: 1) the average number of steps needed to reach a goal, and 2) the average cumulative reward. In each simulation, these measurements are taken per parameter combination, for 3000 epochs in a run, averaged over three repetitions for the 10 mazes (30 runs in total). 

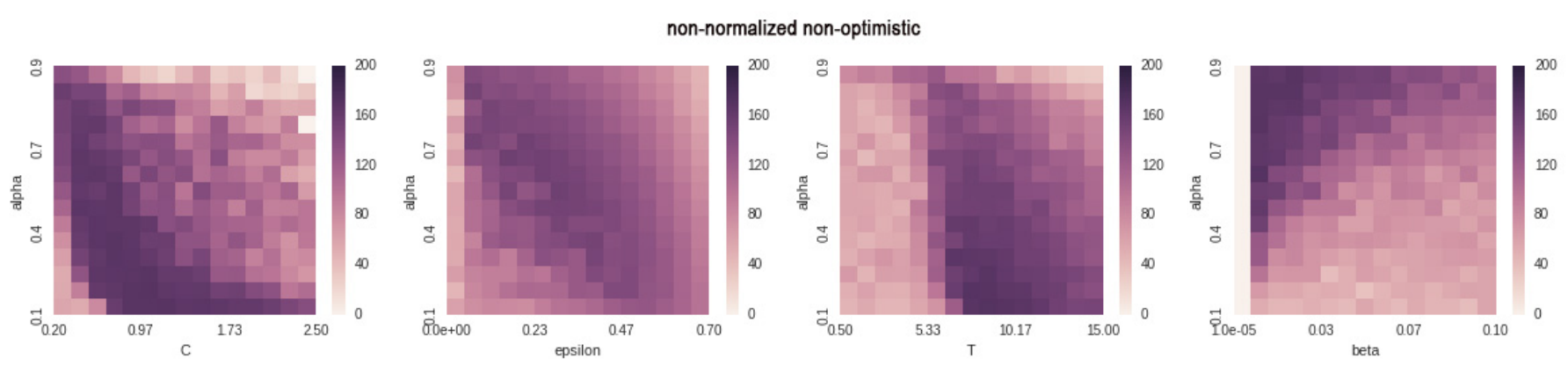

non-normalized optimistic
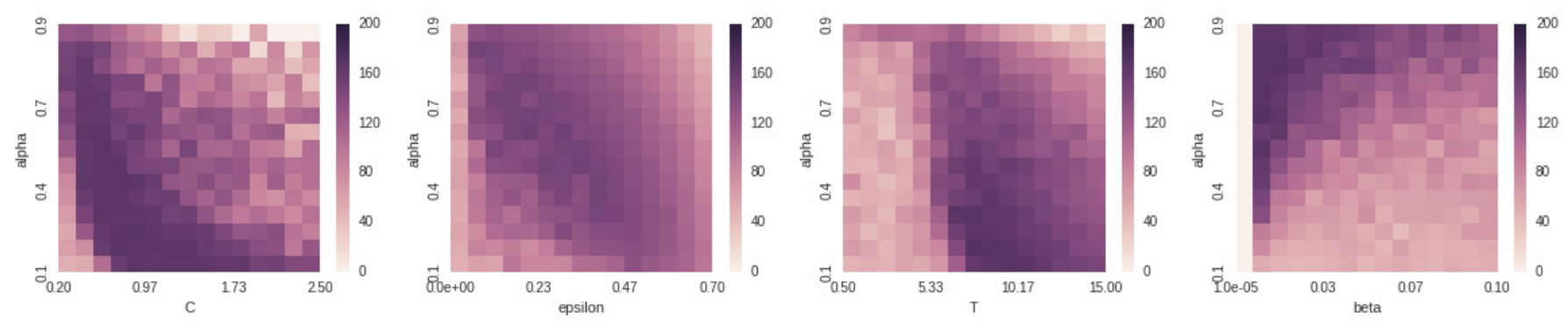

Fig. 2. Heatmaps for the experiment with the unnormalized reward function. The average reward sum intake is shown as a function of parameter combinations ( $x$ and $y$ axes) for the four different exploration strategies combined with Q-learning.

UCB-1
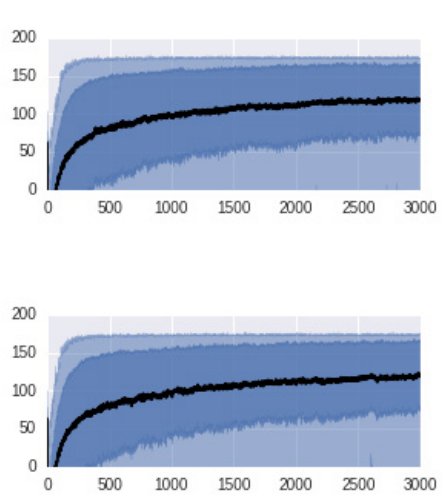

e-greedy

softmax

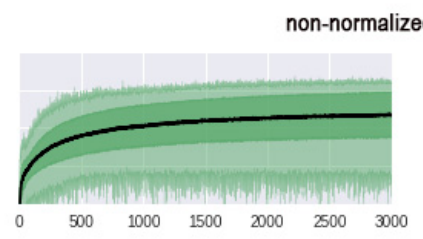

non-normalized optimistic

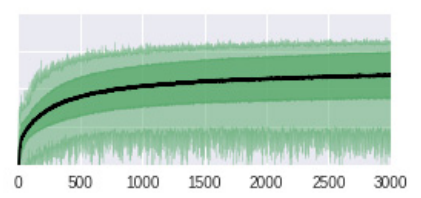

pursuit
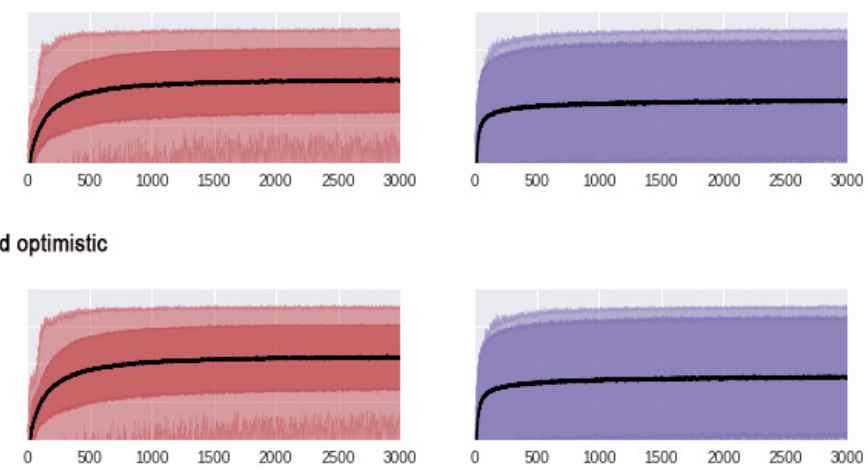

Fig. 3. The learning curves for the four different exploration strategies for the unnormalized reward function. The black line shows the average reward sum obtained for all parameter combinations. The first contour area represents the standard deviation, the second contour area represents the minimum and maximum score for all parameters for the given epoch.

We created a plot per parameter combination and exploration strategy, for which the average obtained reward sums for the last 100 epochs are computed. The resulting heatmaps for the unnormalized reward function are shown in Figure 2.

Figure 2 shows similar results of optimistic and nonoptimistic initialized Q-functions. The heatmaps of pursuit shows that this method requires small values for the learning rate $\beta$. The heatmap for UCB-1 shows that good parameter combinations for $C$ and the learning rate $\alpha$ are negatively correlated. For $\epsilon$-greedy, the best parameter combinations between $\alpha$ and $\epsilon$ are also negatively correlated.

Figure 3 shows the learning curves for all tested parameter combinations of the four exploration strategies. The upper line shows the average reward sum intake of the best parameter combination, whereas the black line shows the average over all tested parameter combinations. If we compare the graphs of the different exploration strategies, it can be seen that $\epsilon$ greedy has the slowest performance increase and thus it needs more time to converge to its optimal performance. Pursuit performs worst when we look at the average results of all 

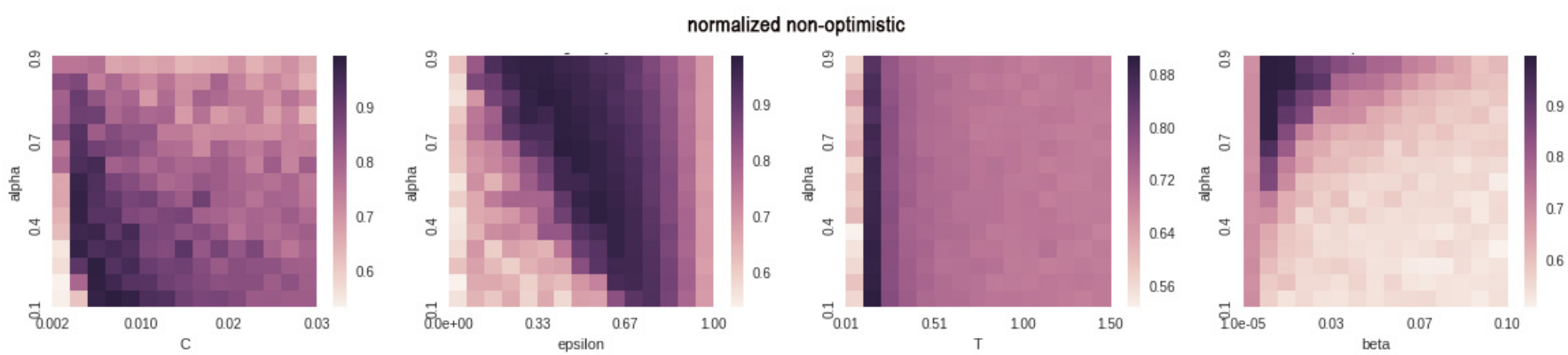

normalized optimistic
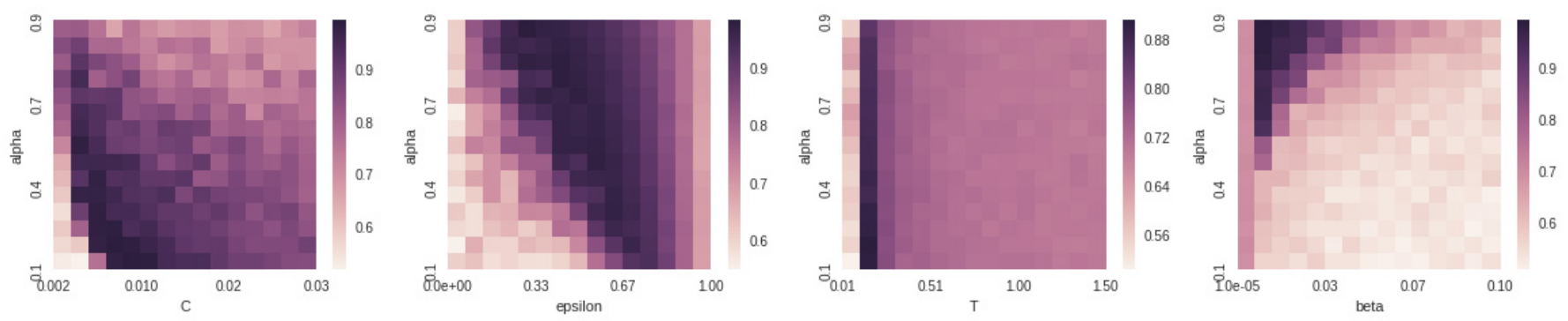

Fig. 4. Heatmaps for the experiment with the normalized reward function. The average reward sum intake is shown as a function of parameter combinations ( $\mathrm{x}$ and $\mathrm{y}$ axes) for the four different exploration strategies combined with Q-learning.

UCB-1
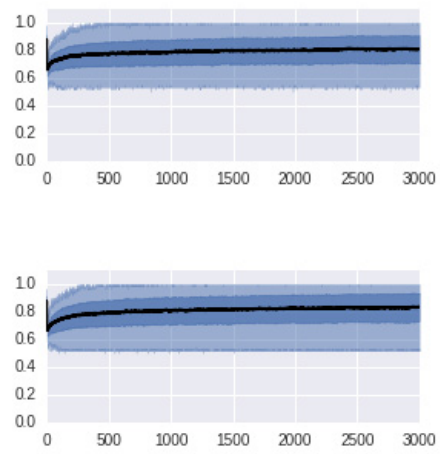

e-greedy

softmax

pursuit
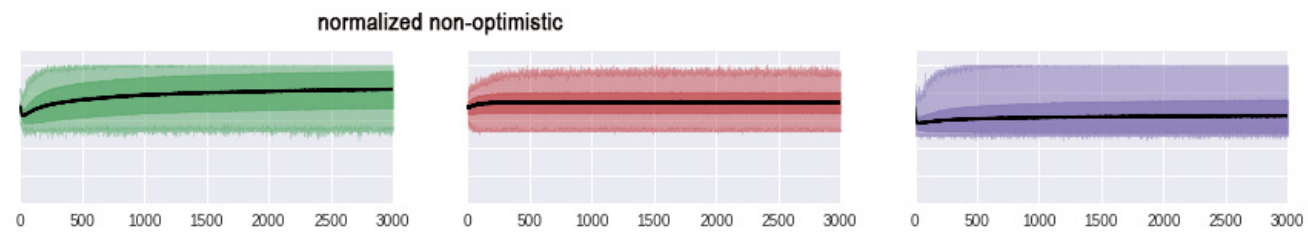

normalized optimistic
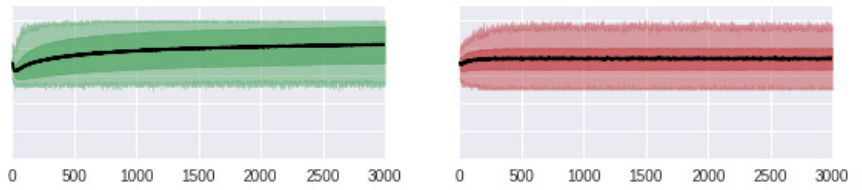

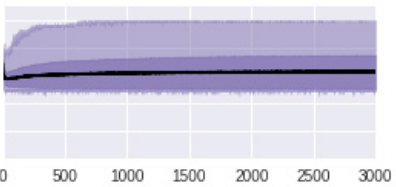

Fig. 5. The learning curves for the four different exploration strategies for the normalized reward function. The black line shows the average reward sum obtained for all parameter combinations. The first contour area represents the standard deviation, the second contour area represents the minimum and maximum score for all parameters for the given epoch.

tested parameter combinations, although it converges fastest.

Figure 4 also shows few differences between optimistic and non-optimistic initialized Q-functions when the normalized reward function is used. The parameter search spaces of UCB-1 and $\epsilon$-greedy are again well-structured, but softmax and pursuit are much more dependent on specific parameter combination settings. The heatmap of pursuit shows again that this method requires very small values for the parameter $\beta$. Softmax only performs very well for a very specific value of the temperature parameter.

Figure 5 shows the learning curves for the four exploration strategies when the normalized reward function is used. With this reward function, there is a less clear difference in the learning processes of the different exploration strategies. Softmax and pursuit perform worse than UCB-1 and $\epsilon$-greedy when we look at the average results of all tested parameter combinations. In the first epochs, the runs are much longer for the normalized reward function compared to the unnormalized 
reward function, because the agent does not learn from (small) negative rewards to unlearn repeating the same actions in the beginning.

The best found parameters for all exploration strategies with the four different experimental setups are shown in Table III.

TABLE III

OPTIMAL PARAMETERS FOR EACH TYPE OF EXPERIMENT

\begin{tabular}{ccc|cc|cc|cc} 
& \multicolumn{2}{c}{$\mathrm{O} / \mathrm{N}$} & \multicolumn{2}{c}{$\neg \mathrm{O} / \mathrm{N}$} & \multicolumn{2}{c}{$\mathrm{O} / \neg \mathrm{N}$} & \multicolumn{2}{c}{$\neg \mathrm{O} / \neg \mathrm{N}$} \\
\hline UCB-1 & $\alpha$ & $C$ & $\alpha$ & $C$ & $\alpha$ & $C$ & $\alpha$ & $C$ \\
\cline { 2 - 10 } & .16 & .007 & .1 & .007 & .16 & .69 & .1 & 1.19 \\
\hline \multirow{2}{*}{-greedy } & $\alpha$ & $\epsilon$ & $\alpha$ & $\epsilon$ & $\alpha$ & $\epsilon$ & $\alpha$ & $\epsilon$ \\
\cline { 2 - 9 } & .95 & .29 & .83 & .36 & .59 & .20 & .65 & .15 \\
\hline Softmax & $\alpha$ & $T$ & $\alpha$ & $T$ & $\alpha$ & $T$ & $\alpha$ & $T$ \\
\cline { 2 - 9 } & .16 & .12 & .1 & .12 & .1 & 8.79 & .1 & 8.79 \\
\hline Pursuit & $\alpha$ & $\beta$ & $\alpha$ & $\beta$ & $\alpha$ & $\beta$ & $\alpha$ & $\beta$ \\
\cline { 2 - 9 } & .95 & .007 & .95 & .007 & .65 & .007 & .59 & .007 \\
\hline
\end{tabular}

After finding the best parameters for each exploration strategy, we performed test runs on new random mazes of sizes $10 \times 10$ and $20 \times 20$. We calculate the average reward sums obtained in 30 runs for each strategy, and rank them. The average reward sums obtained in the $10 \times 10$ mazes can be seen in Table IV. The table shows that softmax consistently performs best, and in most cases significantly outperforms all other exploration strategies. In general, all methods very often find the optimal goal state, but $\epsilon$-greedy performs worst. The table also shows that optimistic initialization often helps the different exploration strategies in the case of the normalized reward function. In this case, softmax always converges to finding the optimal goal state.

TABLE IV

AVERAGE REWARD SUM DURING LAST 100 EPOCHS FOR 10X10 MAZES, HIGHER IS BETTER. 'NORM.' = NORMALIZED, 'OPT.' = OPTIMISTIC.

\begin{tabular}{|c|c|c|c|c|c|c|}
\hline norm. & opt. & UCB-1 & $\epsilon$-greedy & pursuit & softmax & \\
\hline \multirow{6}{*}{ yes } & \multirow{3}{*}{ yes } & 0.989 & 0.974 & 0.998 & 1.000 & $\mu$ \\
\hline & & 0.03 & 0.06 & 0.01 & 0.00 & $\sigma$ \\
\hline & & 3 & 4 & 2 & 1 & rank \\
\hline & \multirow{3}{*}{ no } & 0.961 & 0.980 & 0.997 & 0.998 & $\mu$ \\
\hline & & 0.08 & 0.05 & 0.01 & 0.01 & $\sigma$ \\
\hline & & 4 & 3 & 2 & 1 & rank \\
\hline \multirow{6}{*}{ no } & \multirow{3}{*}{ yes } & 171 & 157 & 174 & 177 & $\mu$ \\
\hline & & 10.7 & 32.4 & 8.0 & 4.7 & $\sigma$ \\
\hline & & 3 & 4 & 2 & 1 & rank \\
\hline & \multirow{3}{*}{ no } & 173 & 158 & 174 & 177 & $\mu$ \\
\hline & & 7.0 & 22.1 & 9.8 & 5.7 & $\sigma$ \\
\hline & & 3 & 4 & 2 & 1 & rank \\
\hline
\end{tabular}

Table $\mathrm{V}$ shows the average number of steps needed for the different methods in the $10 \times 10$ maze. It clearly shows that most methods need less steps when the unnormalized reward function is used. The $\epsilon$-greedy strategy needs a lot of steps to reach the goal in case of the normalized reward function, although it still finds the optimal goal state in most cases as Table IV shows. The reason is probably that the agent learned a repetitive behavior in one or more states, such as colliding against a blocked state from which it can only escape due to the stochasticity in the transition function.

TABLE V

AVERAGE NUMBER OF STEPS NEEDED IN $10 \times 10$ MAZES TO REACH A GOAL. 'NORM.' = NORMALIZED, 'OPT.' = OPTIMISTIC

\begin{tabular}{cccc|c|c|c|c} 
norm. & opt. & UCB-1 & $\epsilon$-greedy & pursuit & softmax & \\
\hline \multirow{4}{*}{ yes } & \multirow{2}{*}{ yes } & 29 & 83 & 33 & 22 & $\mu$ \\
& & 5.0 & 66.9 & 11.2 & 3.2 & $\sigma$ \\
\cline { 2 - 7 } & \multirow{2}{*}{ no } & 27 & 59 & 36 & 47 & $\mu$ \\
& & 5.6 & 37.7 & 14.4 & 19.3 & $\sigma$ \\
\hline \multirow{4}{*}{ no } & \multirow{2}{*}{ yes } & 29 & 31 & 26 & 23 & $\mu$ \\
\cline { 2 - 7 } & & 6.3 & 12.2 & 9.5 & 3.5 & $\sigma$ \\
& \multirow{2}{*}{ no } & 27 & 30 & 26 & 23 & $\mu$ \\
& & 5.8 & 10.4 & 7.1 & 3.7 & $\sigma$ \\
\hline
\end{tabular}

Table VI shows the results of the exploration methods with the same found optimal parameters as before on $20 \times 20$ mazes. Most methods now fail to find the optimal goal state. For the unnormalized reward function UCB-1 performs best. For the normalized reward function, only softmax with optimistic initialization is able to find the optimal goal state in more than $40 \%$ of the cases. Again $\epsilon$-greedy performs worst and is never able to find the optimal goal state. The results show that the larger maze problem is much more difficult for the different exploration strategies. The optimal goal state is further away from the initial position of the agent, and it becomes more likely to converge to a policy that navigates to one of the suboptimal goal states.

TABLE VI

AVERAGE REWARD SUM DURING LAST 100 EPOCHS FOR 20X20 MAZES, HIGHER IS BETTER. 'NORM.' = NORMALIZED, 'OPT.' = OPTIMISTIC.

\begin{tabular}{|c|c|c|c|c|c|c|}
\hline norm. & opt. & UCB-1 & $\epsilon$-greedy & pursuit & softmax & \\
\hline \multirow{6}{*}{ yes } & \multirow{3}{*}{ yes } & 0.500 & 0.500 & 0.516 & 0.716 & $\mu$ \\
\hline & & 0.00 & 0.00 & 0.05 & 0.18 & $\sigma$ \\
\hline & & 3 & 3 & 2 & 1 & rank \\
\hline & \multirow{3}{*}{ no } & 0.500 & 0.500 & 0.517 & 0.500 & $\mu$ \\
\hline & & 0.00 & 0.00 & 0.05 & 0.00 & $\sigma$ \\
\hline & & 2 & 2 & 1 & 2 & rank \\
\hline \multirow{6}{*}{ no } & \multirow{3}{*}{ yes } & 62 & -2 & 21 & 18 & $\mu$ \\
\hline & & 11.4 & 16.7 & 36.0 & 3.8 & $\sigma$ \\
\hline & & 1 & 4 & 2 & 3 & rank \\
\hline & \multirow{3}{*}{ no } & 42 & -2 & 14 & 18 & $\mu$ \\
\hline & & 40.9 & 16.7 & 23.4 & 3.5 & $\sigma$ \\
\hline & & 1 & 4 & 3 & 2 & rank \\
\hline
\end{tabular}

\section{CONCLUSION}

This paper described a study on exploration strategies for Q-learning in stochastic random mazes that have one optimal goal state and two suboptimal goal states. From the range of possible exploration/exploitation techniques for Q-learning, we focused on the undirected strategies: softmax, $\epsilon$-greedy, pursuit, and compared them to the directed exploration strategy UCB-1. The results show that softmax or Boltzmann exploration outperforms the other strategies, although it is harder to tune its parameters. The easiest techniques to tune 
are $\epsilon$-greedy and UCB-1, but $\epsilon$-greedy performs worst of all exploration strategies. The results also show that Q-learning with the different exploration strategies has severe problems in finding optimal policies for a larger maze problem of size $20 \times 20$. This means that the considered problem is quite difficult for model-free reinforcement learning algorithms such as Q-learning. UCB-1 performs well for experiments with unnormalized reward functions, where there is a penalty for every step not directly going to a goal state. UCB-1 performs worse when the reward function is normalized, even though according to literature, this is the range in which the upper bound of this exploration strategy is proven.

In future work, we want to examine if it is possible to create exploration strategies for Q-learning that can consistently find the optimal goal state for larger maze problem without being distracted by the absorbing suboptimal goal states. Furthermore, we want to study the effects of having different types of mazes with more or with less goals, and with dynamic placement of both goals and the agent.

\section{REFERENCES}

[1] R. S. Sutton and A. G. Barto, Reinforcement learning: An introduction. MIT press, 1998

[2] M. A. Wiering and M. van Otterlo, Reinforcement learning: State-ofthe-art. Springer, 2012.

[3] S. B. Thrun, "The role of exploration in learning control," Handbook of intelligent control: Neural, fuzzy and adaptive approaches, 1992.

[4] S. Yahyaa, "Explorations in reinforcement learning: Online action selection and online value function approximation," Ph.D. dissertation, Free University of Brussels, 2015.

[5] M. A. Wiering, "Explorations in efficient reinforcement learning," Ph.D. dissertation, University of Amsterdam, 1999.

[6] C. J. C. H. Watkins, "Learning from delayed rewards," Ph.D. dissertation, King's College, 1989.

[7] S. B. Thrun, "Efficient exploration in reinforcement learning," Tech. Rep., 1992.

[8] J. Vermorel and M. Mohri, "Multi-armed bandit algorithms and empirical evaluation," in European conference on machine learning. Springer, 2005, pp. 437-448.

[9] M. Wiering and J. Schmidhuber, "Efficient model-based exploration," in Proceedings of the Fifth International Conference on Simulation of Adaptive Behavior (SAB98), 1998, pp. 223-228.
[10] M. C. Mozer and J. Bachrach, "Discovering the structure of a reactive environment by exploration," Neural computation, vol. 2, no. 4, pp. 447457, 1990.

[11] S. D. Whitehead and D. H. Ballard, "Learning to perceive and act by trial and error," Machine Learning, vol. 7, no. 1, pp. 45-83, 1991.

[12] R. S. Sutton, "Integrated architectures for learning, planning, and reacting based on approximating dynamic programming," in Proceedings of the seventh international conference on machine learning, 1990, pp. 216-224.

[13] M. Sato, K. Abe, and H. Takeda, "Learning control of finite Markov chains with an explicit trade-off between estimation and control," IEEE Transactions on Systems, Man, and Cybernetics, vol. 18, no. 5, pp. 677684, 1988.

[14] J. Schmidhuber, "Adaptive confidence and adaptive curiosity," in Institut fur Informatik, Technische Universitat Munchen, 1991.

[15] S. B. Thrun and K. Möller, "Active exploration in dynamic environments," in Advances in neural information processing systems, 1992 pp. 531-538.

[16] P. Auer, N. Cesa-Bianchi, and P. Fischer, "Finite-time analysis of the multiarmed bandit problem," Machine Learning, vol. 47, no. 2-3, pp. 235-256, 2002.

[17] V. Kuleshov and D. Precup, "Algorithms for multi-armed bandit problems," arXiv preprint arXiv:1402.6028, 2014.

[18] K. Saito, A. Notsu, and K. Honda, "Discounted UCB1-tuned for QLearning," in Soft Computing and Intelligent Systems (SCIS), 2014 Joint 7th International Conference on and Advanced Intelligent Systems (ISIS), 15th International Symposium on. IEEE, 2014, pp. 966-970.

[19] J. Kennedy, Particle swarm optimization, ser. Encyclopedia of machine learning. Springer, 2011, pp. 760-766.

[20] L. P. Kaelbling, M. L. Littman, and A. W. Moore, "Reinforcement learning: A survey," Journal of artificial intelligence research, vol. 4, pp. 237-285, 1996.

[21] R. E. Bellman, Dynamic Programming. Courier Dover Publications, 1957.

[22] R. S. Sutton, "Learning to predict by the methods of temporal differences," Machine Learning, vol. 3, no. 1, pp. 9-44, 1988.

[23] T. Jaakkola, M. I. Jordan, and S. P. Singh, "On the convergence of stochastic iterative dynamic programming algorithms," Neural computation, vol. 6, no. 6, pp. 1185-1201, 1994.

[24] R. Bellman, "A Markovian decision process," Journal of Mathematics and Mechanics 6, 1957.

[25] R. A. Howard, Dynamic Programming and Markov Processes. Technology Press of Massachusetts Institute of Technology, 1960.

[26] A. G. Barto, S. J. Bradtke, and S. P. Singh, Real-time learning and control using asynchronous dynamic programming. University of Massachusetts at Amherst, Department of Computer and Information Science, 1991. 